\title{
Imbibition of wheat seeds: Application of image analysis**
}

\author{
Jakub Lev* and Jiř Blahovec \\ Faculty of Engineering, Department of Physics, Czech University of Life Sciences Prague, \\ Kamýcká 129, 16500 Prague 6 - Suchdol, Czech Republic \\ Received February 15, 2017; accepted July 7, 2017
}

\begin{abstract}
Image analysis is widely used for monitoring seeds during germination, and it is often the final phase of germination that is subjected to the greatest attention. However, the initial phase of germination (the so-called imbibition) also exhibits interesting behaviour. This work shows that image analysis has significant potential in the imbibition. Herein, a total of 120 seeds were analysed during germination tests, and information about seed size and shape was stored and analysed. It was found that the imbibition can be divided into two newly defined parts. The first one ('abrupt imbibition') consists mainly of the swelling of the seed embryo part and lasts approximately one hour. The second one, referred to as 'main imbibition', consists mainly of spatial expansion caused by imbibition in the other parts of the seed. The results presented are supported by the development of seed cross area and shape parameters, and by direct observation.

Keywords: seed cross area, shape parameters, imbibition stages, embryo development
\end{abstract}

\section{INTRODUCTION}

Wheat (Triticum aestivum L.) is one of the oldest cultivated crops. Indeed, it is the most important food crop in the world. Its germination represents one of the key developmental processes in the plant growth which affects its subsequent grain yield (Podleśna et al., 2015; Kovalyshyn, 2016). Wheat has been in the focus of seed research for many decades, as was summarized in recent papers, for example, Finch-Savage and Leubner-Metzger (2006); Rathjen et al. (2009). Classic methods of assessment, including standard germination tests, have been gradually improved. Image analysis, which is capable of detecting even the smallest changes of the seed dimensions during modified germination tests, has started to play an important part in this process (Dell' Aguila, 2005, 2006, 2009; Dell'

*Corresponding author e-mail: jlev@tf.czu.cz

**This work was supported by the Internal Grant Agency of the Czech University of Life Sciences Prague, Project No. 20163001, (2016).
Aguila et al., 2000; Jossen et al., 2010). The application of image analysis provides much more information about the germination process. Moreover, it is considerably less time-consuming for technicians to undertake, compared to classic tests (Blahovec and Lahodová, 2015a,b; Granitto et al., 2002).

The major advantage of image analysis is the easy determination of dimensional changes in time without any manipulation of the germinated seeds (Tanabata et al., 2012). Using this method, the dimensional inspection of the germinated can be so frequent that the obtained time scans are almost continuous. seed dimensions can be assessed via shape analysis, for example, by the use of characteristic dimensions (Dell' Aguila, 2005; Gegas et al., 2010; Peco et al., 2003; Shouche et al., 2001).

Seed germination is presented as a three-step process (Bewley, 1997; Weitbrecht et al., 2011), in which phases I - III are characterized by increasing seed mass, mainly due to water absorption (Kornarzyński et al., 2002; Resio et al., 2006; Ünal et al., 2013). The first stage, which is distinguished by an initial rapid absorption of water, is referred to as 'early imbibition'. For wheat, this takes about 5-7 h (Abenavoli et al., 2006; Dell'Aquila, 2006; Harb, 2013; Rathjen et al., 2009). This is followed by phase II, in which water absorption is strongly limited, thus also minimizing the seed increase in volume and mass. The length of phase II varies greatly, depending on the seed properties, history (Rathjen, 2009) and degree of dormancy. Phase II concludes with a new increase in the seed's moisture content and mass. This behaviour indicates the start of phase

(C) 2017 Institute of Agrophysics, Polish Academy of Sciences 
III, termed 'proper germination' (Rathjen, 2009). The total increase in mass in phases I - III is about $100 \%$, and slightly more than one half of that is achieved in phase I.

This paper focuses on a meticulous shape analysis of wheat imbibition in phase I and partly in phase II, with the aim of giving a more detailed description of the structure of imbibition in phase I.

\section{MATERIALS AND METHODS}

The wheat seeds were monitored using a specific laboratory setup in the course of a germination test. The particular laboratory setup allows the precise and automatic detection of seed cross area and shape. It consists of a still camera (Canon 450D with Canon EFS 18 - 55 mm lens), a tripod, an illumination LED panel, and a glass vessel for the germination test. The camera was controlled via computer using DSLR Remote Pro for Windows version 2.7.2 (Breeze Systems, United Kingdom). Images from the camera were stored in a hard disk in RAW format. The focal length was set to the top surface of the seeds. The LED panel was controlled via computer to illuminate the specimens for $3 \mathrm{~s}$ at the moment when the picture was taken. The interval between each photograph was $120 \mathrm{~s}$. The whole setup was placed in a dark box. This setup was already introduced by Lev et al. (2016).

Winter wheat (variety: Tosca, supplier: Selgen a.s.) harvested in 2014 was used for the experiments. The moisture content (wet basic) of the seeds was $8-9 \%$. Data was collected during six germination tests, and the experiments took place in January and February 2016. The germination tests were performed on agar $0.8 \%$ in a glass vessel covered by a thin acrylic glass plate. In each experiment, 20 seeds were monitored. The seeds, turned with the germ on the left, were evenly placed on the agar with a $318.5 \mathrm{~mm}^{2}$ scale next to them. The scale made it possible to determinate the true sizes of the seeds, and was made from a small plastic plate.

Each germination test continued either until all seeds successfully germinated, or for a maximum of 7 days. All in all, 120 seeds were monitored, and only one seed did not germinate. The temperature in the laboratory was maintained at $20-22^{\circ} \mathrm{C}$ throughout the course of the experiments. The humidity and pressure in the laboratory were $30-40 \%$ and $95.4-100.1 \mathrm{kPa}$, respectively.

The image processing and data analysis were performed after the termination of the experiments. For this purpose, the programming language Python 2.7 was used, together with supporting libraries (OpenCV 2.4.8, NumPy 1.8.2, and Matplotlib 1.3.1). Image processing occurred as follows:

- Conversion to grayscale, followed by conversion to a binary image. For all images, blue channel and a threshold value of 140 were used. This was possible because identical light conditions were ensured during all experiments.
- Application of an erosion-dilation filter for noise reduction.

- Outline definition and determination of seed cross areas. The cross area in $\mathrm{mm}^{2}$ was determined. For this purpose, the area of one pixel was determined by first referring to an object with a known area (the scale).

- Labelling of individual seed regions and their orientation so that the major axis of each seed was parallel with the $x$-axis.

- Determination of the major seed size (length) and the minor seed size (width).

- Determination of normalized central moments.

The angle of orientation of each seed outline was calculated as follows (Shouche et al., 2001):

$$
\theta=\frac{1}{2} \arctan \left(\frac{2 \mu_{1,1}}{\mu_{2,0}-\mu_{0,2}}\right),
$$

where: $\theta$ is the angle of the seed outline major axis and $\mu_{1,1}, \mu_{2,0}$ and $\mu_{0,2}$ are central moments (Gonzalez and Woods, 2002; Hu, 1962) defined by the following equation:

$$
\mu_{p, q}=\sum_{x} \sum_{y}\left(x-x_{c}\right)^{p}\left(y-y_{c}\right)^{q} f(x, y),
$$

where: $p, q$ are natural numbers, $x_{c}, y_{c}$ are the components of the centroid, and $f(x, y)$ is a digital image. The last step of the image processing was the determination of the normalized central moment $\eta_{3,0}(\mathrm{Hu}, 1962$; Gonzalez and Woods, 2002):

$$
\eta_{3,0}=\frac{\mu_{3,0}}{m_{0,0}^{\frac{3}{2}+1}},
$$

where: $m_{0,0}$ is the outline area. It should be noted here that the central moments $\mu_{3,0}$ were calculated after the normalized rotation of the seed outlines.

The development of several parameters (seed cross area, seed length, and seed width) was evaluated using a time derivative, calculated numerically. Experimental data was sequentially fitted by straight lines in order to estimate the course of the first derivative. The straight lines parameters were determined by the application of the least squares method, always in an interval containing twelve measured values. The interval was moved after one measured value, and the estimate of the derivative in the middle of the interval expressed the slope of the straight line found. The following parameters were calculated: the seed area rate (SAR), the seed length rate (SLR), and the seed width rate (SWR).

For the purpose of data comparison, the SAR, SLR and SWR were converted using the following formula:

$$
y_{n}=\frac{y}{Y_{r}},
$$


where: $y_{n}$ is the normalized data, $y$ is the input data, and $Y_{r}$ is the normalized value. This value is defined as a mean value of the input data during the first $2 \mathrm{~h}$. For the purpose of the $\eta_{3,0}$ comparison, the following formula was used:

$$
n_{3,0}=\eta_{3,0}-\eta 0_{3,0},
$$

where: $n_{3,0}$ is the moved normalized central moment and $\eta 0_{3,0}$ is the initial normalized central moment.

During the data analysis process, the selected data were fitted (using the least squares method) by linear, polynomial, or exponential functions. The data SAR, SLR, and SWR were compared by the Spearman correlation coefficient.

\section{RESULTS AND DISCUSSION}

The size change of the seed (swelling) depends on water content. The important parameter, which was obtained in this research, was the seed cross area. Whereas the seed mass development is characterized by quick increase in the first germination phase (early imbibition) and only a small increase in the second germination phase, the time course of the seed cross area grows distinctly in both phases. This is caused by the decrease in seed density during the second germination phase (Blahovec and Lahodová, 2015b). One example of seed cross area development is shown in Fig. 1a. The seed was labelled ' $m 3$ 19' for the purpose of this research. If it is assumed that the length of the first germination phase is approximately 5-7 h (Abenavoli et al., 2006; Dell'Aquila, 2006; Rathjen et al., 2009), then the discussed figure should contain the whole of the first and part of the second germination phases.

The seed cross area development in Fig. 1a corresponds to previous research (Blahovec and Lahodová, 2015b; Dell'Aguila, 2006; Kruse, 2000). However, the time course of the seed cross area contains a more complicated region at the beginning (approximately up to $2.5 \mathrm{~h}$ ). This region is highlighted with a dashed line, and it can be observed that the time course of the seed cross area changes in character between concave and convex. This implies that the SAR alternately falls and rises. This behaviour is much clearer from part Fig. 1b, where the time course of the SAR is depicted for the same seed. Local extremes of this curve introduce the inflection points on the seed cross area development, which are labelled $\mathrm{P}_{1}$ and $\mathrm{P}_{2}$, respectively.

The behaviour described in the previous paragraph was observed in almost all the samples tested. Indeed, in only two cases (out of the total of 120 tested seeds) such behaviour was seen as different, and it was impossible to detect the points $P_{1}$ and $P_{2}$. The discussed shape of the SAR curve is also apparent in Fig. 2, where the whole data set is presented. Fig. 2 contains the mean values and standard deviations of the normalized SAR. The data was normalized according to Eq. (4).
Both $\mathrm{P}_{1}$ and $\mathrm{P}_{2}$ are located in the first germination phase, as the length of this phase is normally 5-7 $\mathrm{h}$ (Abenavoli et al., 2006; Dell'Aquila, 2006; Rathjen et al., 2009). The average time between the beginning of the imbibition process and points $P_{1}$ and $P_{2}$ is 0.995 and $1.768 \mathrm{~h}$, respectively. The standard deviations are 0.208 and $0.350 \mathrm{~h}$, respectively.
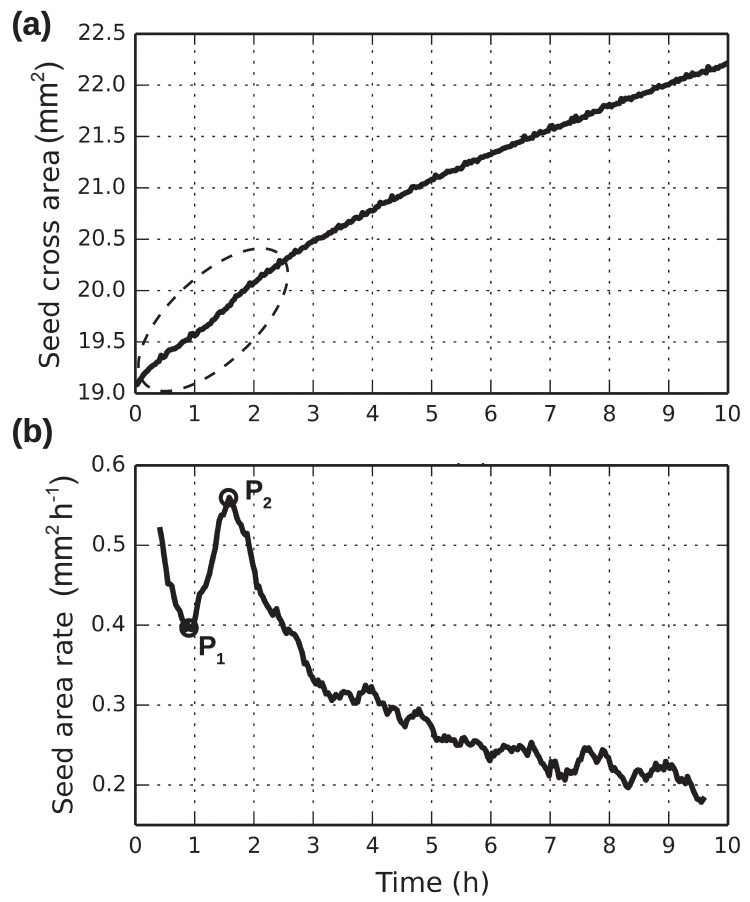

Fig. 1. Example of seed development: a - the time course of the seed cross area. The region which is the object of interest is highlighted by the dashed line; $b$ - the time course of the SAR; points $\mathrm{P}_{1}$ and $\mathrm{P}_{2}$ are plotted in the graph; $\mathrm{P}_{1}$ at $0.87 \mathrm{~h}$ and $\mathrm{P}_{2}$ at $1.62 \mathrm{~h}$.

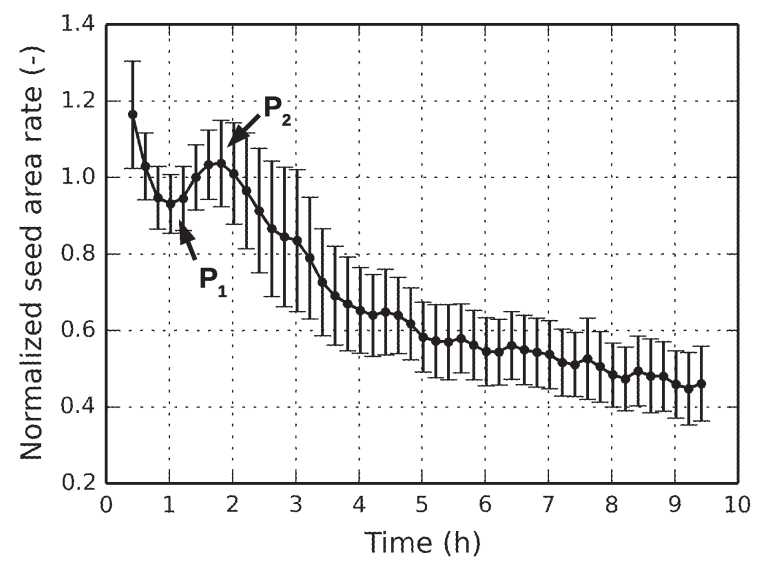

Fig. 2. Development of the normalized SAR mean values and standard deviations with points $\mathrm{P}_{1}$ and $\mathrm{P}_{2}$. Only every sixth value is plotted. The data was normalized according to Eq. (4). 
It has also been noted that the seed length and width have different time courses. Whereas the time course of the seed width has an approximately linear character, the time course of the seed length has a non-linear character and resembles the seed cross area development.

Figure 3 depicts the mean values of the SLR and the SWR, together with their standard deviations (the data was first normalized according to Eq. (4)). The SLR decreases significantly during the imbibition process. The drop is approximately 0.87 during the first $6 \mathrm{~h}$. The behaviour is different in the case of the SWR, with a minor reduction (approximately 0.32 in the first $6 \mathrm{~h}$ ) being visible. However, the decrease is provable with a p-value $<0.0001$ in both cases. The time course of the SLR is very similar to the SAR. Primarily, the initial part of the SLR curve contains similar local extremes (labelled $\mathrm{P}_{1}$ and $\mathrm{P}_{2}$ in the SAR curve).

The Spearman correlation coefficients were calculated between SAR and SLR data, and also between SAR and SWR data (separately for each seed). The average correlation coefficient between SAR and SLR is $R=0.79$. The correlation between SAR and SWR is weaker, with the average coefficient amounting to $\mathrm{R}=0.36$. It can, therefore, be deduced that the seed cross area and the SAR development during imbibition are significantly influenced by processes that mainly affect the seed length.

Figure 4 depicts two seeds after $10 \mathrm{~h}$ of imbibition. The actual seed outlines are marked in green. Black, blue, and red marks represent the seed outline in the initial state, after $1 \mathrm{~h}$ of imbibition, and after $2 \mathrm{~h}$ of imbibition, respectively. It is clear that the most significant development in the seed embryo occurs during the first hour of the imbibition process. This behaviour can be observed in both of the exhibited examples, as well as in all of the tested samples. The imbibition process is more uniform during the second hour, and the seed embryo development is much less significant. It seems that the initial stage up to $\mathrm{P}_{1}$ (first hour) is influenced mainly by the development of some parts of the embryo, and the stage between points $\mathrm{P}_{1}$ and $\mathrm{P}_{2}$ (second hour) is a result of swelling in other parts of the seed. After $10 \mathrm{~h}$, the swelling process is apparent throughout the whole seed. Stronger swelling is visible on the side closer to the embryo and around the brush.

The normalized central moment $\eta_{3,0}$ is sensitive to asymmetry along the $y$-axis (seed minor axis). Therefore, it could potentially be used to detect small changes in the shape in the embryo and the brush. One example of the time course of $\eta_{3,0}$ (seed ' $m 3$ 19') is shown in Fig. 5. The significant decrease in $\eta_{3,0}$ is visible during the first hour. This behaviour is likely to result from the swelling of an embryo part. The decrease stops approximately at $1.2 \mathrm{~h}$. The increase in the rest of the graph can imply predominant swelling around the brush, or an increase in seed thickness on the left side.
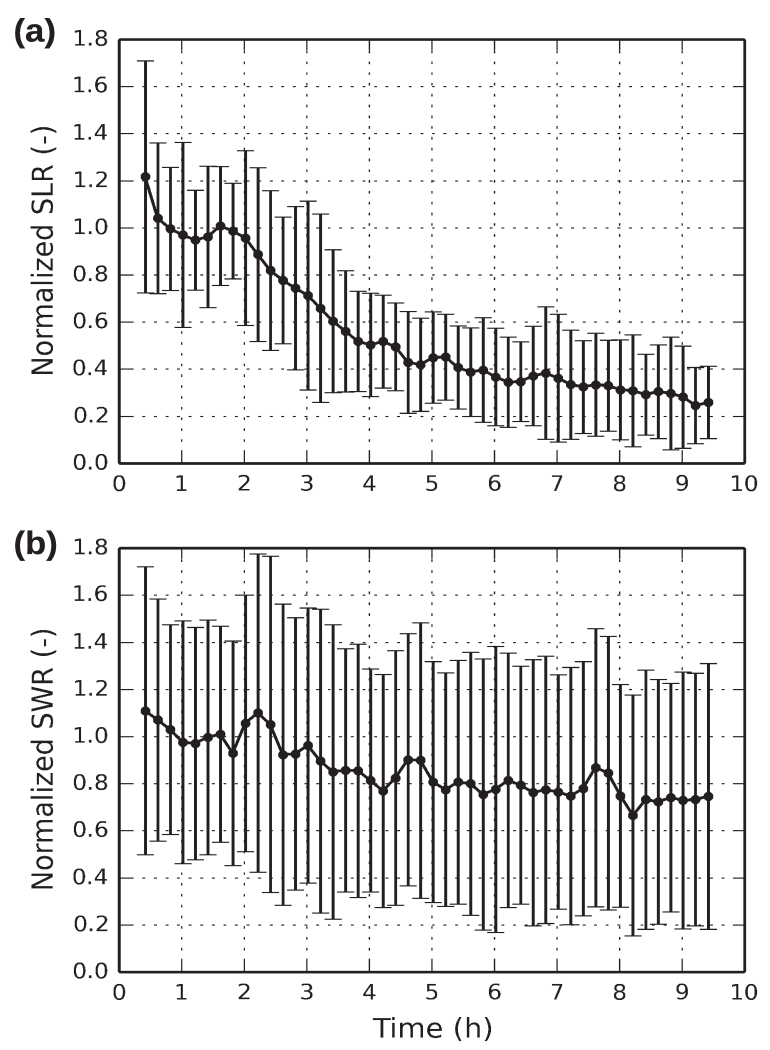

Fig. 3. Development of normalized SLR mean values and standard deviations (a); development of normalized SWR mean values and standard deviations (b). The data was normalized according to Eq. (4) and only every sixth value is plotted in both cases.

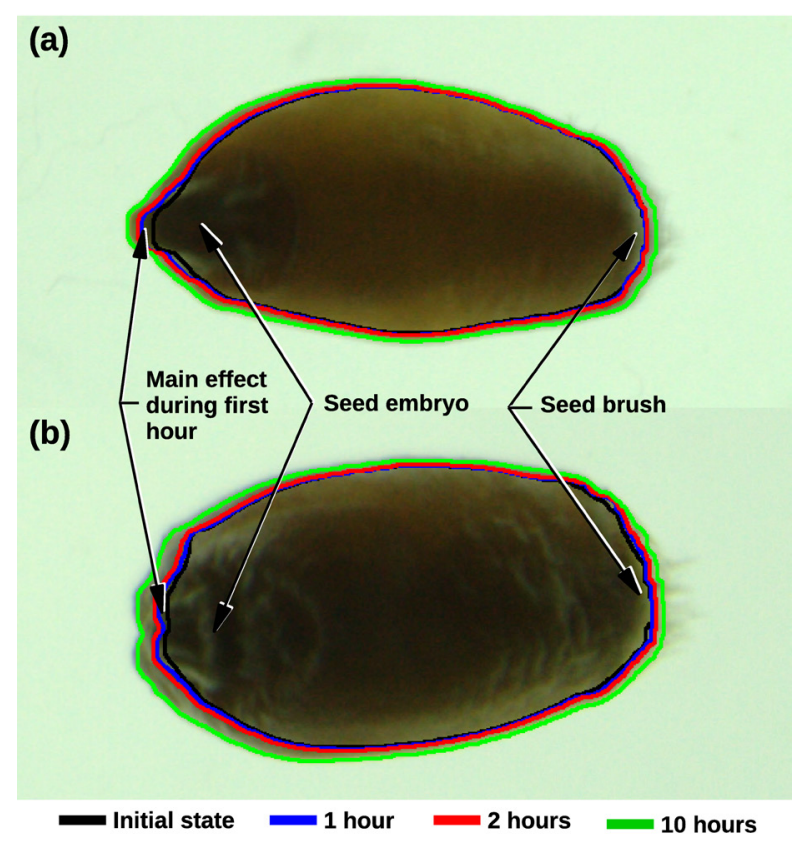

Fig. 4. Two examples of seeds after ten hours of imbibition. The coloured curves represent the seed outlines at different imbibition times. a-seed labelled ' $m 3$ _02', b-seed labelled ' $m 3$ _19'. 


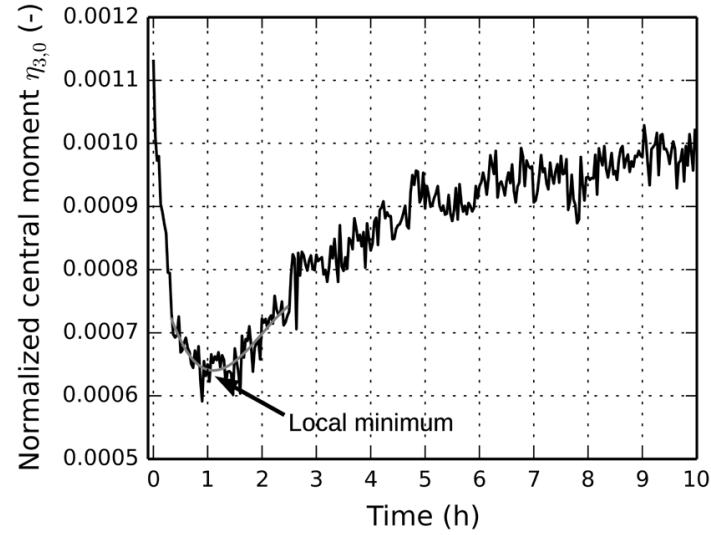

Fig. 5. Development of the normalized central moment of seed ' $\mathrm{m} 3$ _ 19'. The stage from $0.4 \mathrm{~h}$ to $2.5 \mathrm{~h}$ is fitted by a third degree polynomial function the grey curve for the purpose of determining the local minimum.

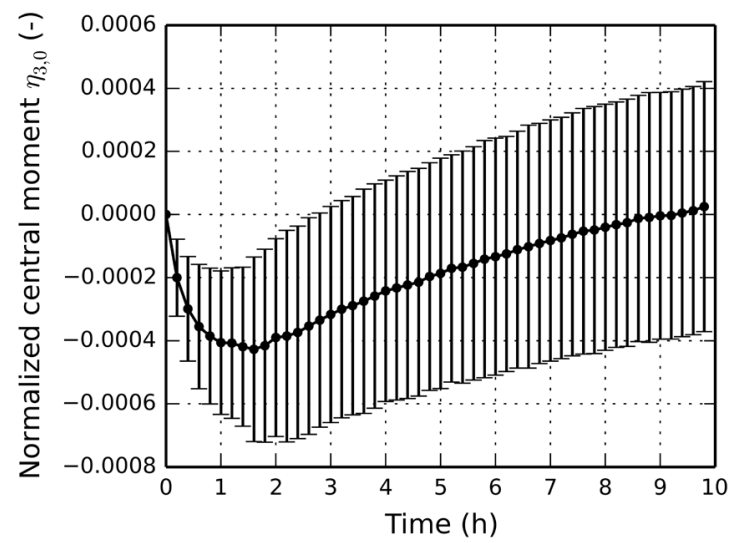

Fig. 6. Development of $\eta_{3,0}$; data as modified using Eq. (5). Mean values and standard deviations are depicted (only every sixth value is plotted).

The local minimum to be seen in Fig. 5 could be connected with the end of the quick embryo swelling (its part). This minimum was labelled $\mathrm{N}_{\mathrm{m}}$, and the point was determined for all tested seeds. The average $\mathrm{N}_{\mathrm{m}}$ time for the set being tested was $1.265 \mathrm{~h}$, with a standard deviation of $0.643 \mathrm{~h}$. The relationship between points $\mathrm{P}_{1}, \mathrm{P}_{2}$, and $\mathrm{N}_{\mathrm{m}}$ was also evaluated: the ratio of $\mathrm{N}_{\mathrm{m}} / \mathrm{P}_{1}$ is larger than 1 , and $\mathrm{N}_{\mathrm{m}} /$ $\mathrm{P}_{2}$ is smaller than 1 (both with a p-value $<0.0001$ ). This means that $\mathrm{N}_{\mathrm{m}}$ is located between $\mathrm{P}_{1}$ and $\mathrm{P}_{2}$. A comparison of all the seeds tested is presented in Fig. 6, with the values modified using Eq. (5). This graph shows that the presented development of the normalized central moment is typical for most seeds.

Rathjen et al. (2009) describe water movement into wheat grains. The authors state that the embryo and scutellum are substantially more hydrated than the other parts of the seed during the whole germination process. Water is mainly located within some parts of the embryo, the scutel- lum, and the coat during early imbibition. As the imbibition process continues, the water enters into other parts of the embryo. However, the endosperm is hydrated much more slowly. The water enters the endosperm primarily through the scutellum, and this process starts after $7 \mathrm{~h}$ of imbibition (Gruwel et al., 2001; Rathjen et al., 2009).

The behaviour observed during the seed imbibition, as presented in this work, is connected with water movement into the seed. The stage from the start of the imbibition process to point $\mathrm{P}_{1}$ is remarkable, being characterized by rapid swelling in the area where the radicle and micropyle are located. The micropyle plays a very important role during this stage because the water enters the seed through it (Gruwel et al., 2002; Rathjen et al., 2009). This short stage (Ia) can be termed 'abrupt imbibition'. The results of our research confirm that, during premature imbibition, water first enters into certain parts of the embryo (probably the radicle), which can be detected via image analysis.

The connection between the Ia stage and embryo development is supported by the following results. Firstly, significant increases in the area close to the seed embryo were observed during the first hour (Fig. 4). Secondly, a good correlation between SAR and SLR $(\mathrm{R}=0.79)$, and, conversely, a poor correlation between SAR and SWR $\mathrm{R}=0.36$ ) were noted. This indicates that SAR development is particularly influenced by processes connected with the embryo and the brush. Thirdly, the initial rapid decrease in the development of $\eta_{3,0}$ can be explained in two ways, i.e. as a significant increase in the seed width near the brush, or as swelling in a small part of the embryo area that had no effect on the seed width (for example, the radicle). The first case was not observed, whereas the second was.

Abrupt imbibition represents only a short part of the whole imbibition process. From point $\mathrm{P}_{1}$, the area increase is the result of the swelling observed throughout the whole seed. This stage could be referred to as "main imbibition'. The main imbibition stage contains two interesting points. The first is the local minimum of $\eta_{3,0}$. As has already been mentioned, $\mathrm{N}_{\mathrm{m}}$ is probably connected with the end of the quick swelling of a part of the embryo. This point can be connected with swelling in the whole embryo and the scutellum (with effect to the seed width) or swelling around the brush. The second point is $\mathrm{P}_{2}$, which represents the maximum swelling speed in the main imbibition stage. This point could be a useful indicator of the imbibition rate.

The imbibition process was divided into two stages in the preceding text. It can be assumed that these stages result from at least two processes. The first process is swelling in the embryo part (abrupt swelling), and the second process is swelling in other seed parts. Abrupt swelling strongly impacts the Ia stage. It can be estimated that the contribution of abrupt swelling to SAR development has an approximately exponential trend and can be described by the following equation: 


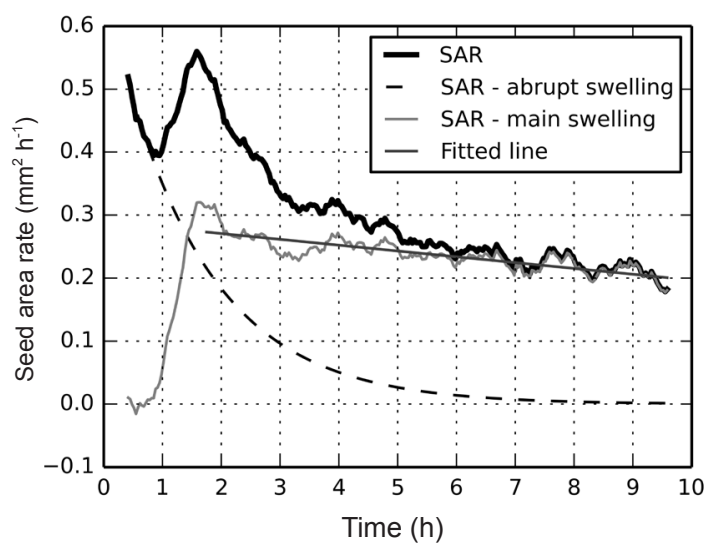

Fig. 7. Estimated contribution of the abrupt swelling process and the main swelling process to SAR. The abrupt swelling contribution is expressed by the dashed curve and the exponential function $\mathrm{SAR}_{\mathrm{a}}=0.666 \exp (-0.645 t)$. The grey curve represents the effect of other seed parts swelling and contains a linear part. This part is fitted by the linear function (the dark line) $y=-0.0092 t+0.29$, $\mathrm{R}^{2}=0.71$. This analysis was done for seed ' $\mathrm{m} 3 \_19$ '.

$$
\mathrm{SAR}_{\mathrm{a}}=\mathrm{A} e^{-\mathrm{B} t}
$$

where: $\mathrm{SAR}_{\mathrm{a}}$ is the contribution to SAR development, A and $\mathrm{B}$ are constants representing seed properties and environment, and $t$ is time.

The SAR development (the black curve) of the seed ' $m 3$ _19' is shown in Fig. 7. The initial SAR data, corresponding to the Ia stage, was fitted to the exponential function Eq. (6), and the probable effect of abrupt swelling in future development was determined. This data is presented by the black dashed curve. The difference between SAR data and the effect of abrupt swelling is depicted by the grey curve in the same figure. This curve mainly represents the effect of the swelling of other seed parts. The resulting curve can be divided into two parts. The first part (approximately $1.7 \mathrm{~h}$ ) is characterized by a quick increase. It seems that water motion inside the seed is irregular enough that the imbibition starts in the radicle (Rathjen et al., 2009). The second part exhibits an approximately linear and slowly decreasing character. This analysis was applicable to 79 of the tested seeds (where Ia was sufficiently long). In all cases, similar behaviour was observed.

\section{CONCLUSIONS}

1. Whereas the first and the second germination phases are well defined by seed mass development, the seed cross area development serves as good indicator of two new stages of the first germination phase.

2. The first stage: Ia (abrupt imbibition) mainly consists of the swelling of the seed embryo part. Our results showed the duration of this stage to be approximately $1 \mathrm{~h}$.
3. The second stage: Ib (main imbibition), starting at approximately this time, mainly consists of a spatial expansion induced by imbibition of the other parts of the seed.

4. Ib contains a characteristic point which represents the highest mean rate of seed swelling in this stage.

5. The two presented new stages can be detected via image analysis.

Conflict of interest: The Authors do not declare conflict of interest.

\section{REFERENCES}

Abenavoli M.R., Cacco G., Sorgonà A., Marabottini R., Paolacci A.R., Ciaffi M., and Badiani M., 2006. The inhibitory effects of coumarin on the germination of durum wheat (Triticum turgidum ssp. durum, CV. Simeto) seeds. J. Chemical Ecology, 32, 489-506.

Bewley J.D., 1997. Seed germination and dormancy, Plant Cell, 9, 1055-1066.

Blahovec J. and Lahodová M., 2015a. Moisture-induced changes of mass and dimension characteristics in some cereal grains. Int. Agrophys., 29, 1-12.

Blahovec J. and Lahodová M., 2015b. Moisture induced changes of volume and density of some cereal seeds. Plant Soil Environ., 61, 43-48.

Dell'Aquila A., 2005. The use of image analysis to monitor the germination of seeds of broccoli (Brassica oleracea) and radish (Raphanus sativus). Annals of Appl. Biol., 146, 545-550.

Dell'Aquila A., 2009. New perspectives for seed germination testing through digital imaging technology. The Open Agriculture J., 3, 37-42.

Dell'Aquila A.D., 2006. Computerized seed imaging: a new tool to evaluate germination quality. Communications in Biometry Crop Sci., 1, 20-31.

Dell'Aquila A., van Eck J.W., and van der Heijden G.W.A.M., 2000. The application of image analysis in monitoring the imbibition process of white cabbage (Brassica oleracea L.) seeds. Seed Sci. Res., 10, 163-169.

Finch-Savage W.E. and Leubner-Metzger G., 2006. Seed dormancy and the control of germination. New Phytologist, $171,501-523$.

Gegas V.C., Nazari A., Griffiths S., Simmonds J., Fish L., Orford S., Sayers L., Doonan J.H., and Snapea J.W., 2010. A genetic framework for grain size and shape variation in wheat. Plant Cell, 22, 1046-1056.

Gonzalez R.C. and Woods R.E., 2002. Digital Image Processing. Prentice Hall, New Jersey, USA.

Granitto P.M., Navone H.D., Verdes P.F., and Ceccatto H.A., 2002. Weed seeds identification by machine vision. Computers Electronics Agric., 33, 91-103.

Gruwel M.L.H., Chatson B., Yin X.S., and Abrams S., 2001. A magnetic resonance study of water uptake in whole barley kernels. Int. J. Food Sci. Technol., 36, 161-168.

Gruwel M.L.H., Yin X.S., Edney M.J., Schroeder S.W., MacGregor A.W., and Abrams S.R., 2002. Barley viability during storage: use of magnetic as a potential tool to study viability loss. J. Agric. Food Chem., 50, 667-676. 
Harb A.M., 2013. Reserve mobilization, total sugars and proteins in germinating seeds of durum wheat (Triticum durum Desf.) under water deficit after short period of imbibition. Jordan J. Biological Sci., 6, 67-72.

Hu M.K., 1962. Visual pattern recognition by moment invariant. IRE Trans. Information Theory IT 8, 179-187.

Joosen R.V.L., Kodde J., Willems L.A.J., Ligterink W., van der Plas L.H.W., and Hilhorst H.W.M., 2010. GERMINATOR: a software package for high-throughput scoring and curve fitting of Arabidopsis seed germination. Plant J., 62, 148-159.

Kornarzynski K., Pietruszewski S., and Lacek R., 2002. Measurement of the water absorption rate in wheat grain. Int. Agrophysics, 16, 33-36.

Kovalyshyn S., 2016. Study of structural changes in the cells of the stimulated seed sprouts. Int. Agrophys., 30, 545-550.

Kruse M., 2000. The effect of moisture content on linear dimensions in cereal seeds measured by image analysis. Seed Sci. Technol., 28, 779-791.

Lev J., Lahodová M., and Blahovec J., 2016. Precise automatic detection of plant seed germination. In: Proc. 6th Int. Conf. Trends in Agricultural Engineering, September 7-9, Prague, Czech Republic.
Peco B., Traba J., Levassor C., Sánchez A.M., and Azcárate F.M., 2003. Seed size, shape and persistence in dry Mediterranean grass and scrublands. Seed Sci. Res., 13, 87-95.

Podleśna A., Gładyszewska B., Podleśny J., and Zgrajka W., 2015. Changes in the germination process and growth of pea in effect of laser seed irradiation. Int. Agrophys., 29, 485-492.

Rathjen J.R., Strounina E.V., and Mares D.J., 2009. Water movement into dormant and non-dormant wheat (Triticum aestivum L.) grains. J. Exp. Botany, 60, 1619-1631.

Resio A.C., Aguerre R.J., and Suarez C., 2006. Hydration kinetics of amaranth grain. Food Eng., 72, 247-253.

Shouche S.P., Rastogi R., Bhagwat S.G., and Sainis J.K., 2001. Shape analysis of grains of Indian wheat varieties. Computers Electronics Agric., 33, 55-76.

Tanabata T., Shibaya T., Hori K., Ebana K., and Yano, M., 2012. SmartGrain: High-throughput phenotyping software for measuring seed shape through image analysis. Plant Physiology 160, 1871-1880.

Ünal H., Alpsoy H.C., and Ayhan A., 2013. Effect of the moisture content on the physical properties of bitter gourd seed. Int. Agrophys., 27, 455-461.

Weitbrecht K., Müller K., and Leubner-Metzger G., 2011. First off the mark: early seed germination. J. Exp. Botany, 62, 3289-3309. 